\title{
Investigation of defect formation and electronic transport in microcrystalline silicon deposited by hot wire CVD
}

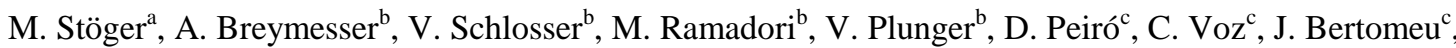 \\ M. Nelhiebel ${ }^{\mathrm{a}}$, P. Schattschneider ${ }^{\mathrm{a}}, \mathrm{J}$ Andreu $^{\mathrm{c}}$ \\ ${ }^{a}$ Institut für Angewandte und Technische Physik, Technical University Wien, A-1040 Wien, Austria \\ ${ }^{\mathrm{b}}$ Institut für Materialphysik, Universität Wien, A-1090 Wien, Austria \\ ${ }^{\mathrm{c} D}$ Departament de Física Aplicada i Electrònica, Universitat de Barcelona, E-08028 Barcelona, Spain
}

\begin{abstract}
We have investigated doped and undoped layers of microcrystalline silicon prepared by hot wire chemical vapour deposition optically, electrically and by means of transmission electron microscopy. Beside needlelike crystals grown perpendicular to the substrate's surface all of the layers contained a noncrystalline phase with a volume fraction between $4 \%$ and $25 \%$. A high oxygen content of several per cent in the porous phase was detected by electron energy loss spectrometry. Deep level transient spectroscopy of the crystals suggests that the concentration of electrically active defects is less than $1 \%$ of the undoped background concentration of typically $10^{17} \mathrm{~cm}^{-3}$. Frequency dependent measurements of the conductance and capacitance perpendicular to the substrate surface showed that a hopping process takes place within the noncrystalline phase parallel to the conduction in the crystals. The parasitic contribution to the electrical circuit arising from the porous phase is believed to be an important loss mechanism in the output of a pin-structured photovoltaic solar cell deposited by hot wire CVD.
\end{abstract}

Keywords: microcrystalline silicon, transmission electron microscopy, deep level transient spectroscopy, nanopotentiometry.

Corresponding author: Viktor Schlosser

Institut für Materialphysik der Universität Wien

Strudlhofgasse 4, A-1090 Wien (Austria)

FAX: (+43 1) 586340813

phone: (+43 1) 586340927

e-mail: viktor.schlosser@univie.ac.at 


\section{Introduction}

In the last years the deposition of polycrystalline silicon on glass substrates has attracted great interest for the preparation of thin film transistors and thin film solar cells. For photovoltaic applications polycrystalline films are believed to combine the advantage of a low temperature deposition technique similar to the preparation of amorphous silicon with the high long term stability known from crystalline silicon solar cells. Recently conversion efficiencies of $8.5 \%$ have been demonstrated for thin amorphous-microcrystalline silicon solar cells on glass substrates [1]. The limited thermal stability of the glass substrate which corresponds to about $550^{\circ} \mathrm{C}$ in the case of borosilicate glass requires deposition techniques at low temperatures. Several methods have been reported which allow the deposition of crystalline silicon layers at temperatures as low as $190^{\circ} \mathrm{C}$ [2]. At this temperatures which are several hundred degrees below the typical growth temperature used for epitaxial silicon deposition the morphology and the electronic quality of the deposited layers are extremely sensitive to the selected deposition parameters. In the present work microcrystalline silicon - $\mu \mathrm{c}-\mathrm{Si}$ - deposited by Hot-Wire Chemical Vapour Deposition (HWCVD), which is sometimes referred to as catalytic CVD, was investigated. Crystalline layer growth has been observed at substrate temperatures even below $190^{\circ} \mathrm{C}$.

\section{Experimental}

Undoped $\mu \mathrm{c}$-Si:H layers were deposited on Corning 7059 glass and on conducting zinc oxide $(\mathrm{ZnO})$ coated glass in one of the chambers of an UHV multichamber setup [3]. The ZnO layer served as a transparent, electrical contact. The process parameters which were varied are (i) the silane to hydrogen ratio, (ii) the process pressure, (iii) the gas flow, (iv) the substrate temperature and (v) the filament temperature. For the formation of a pin-structure diborane was added to the gas flow in the case of $p$-Si deposition and phosphine was added in the case of $n$-Si deposition. For transmission electron microscopy (TEM) and electron energy loss spectrometry (EELS) thin specimen have been prepared by gluing together two pieces of a sample surface to surface thus preventing the thin film from splintering away during abrasion. Thinning was done mechanically and by the use of an ion polishing system. Two types of rectifying structures were prepared: (i) undoped $\mu \mathrm{c}-\mathrm{Si}$ on transparent $\mathrm{ZnO}$ and (ii) a pin-device grown on $\mathrm{ZnO}$. In the first case the thickness of the $\mu \mathrm{c}$-Si film was about $1 \mu \mathrm{m}$. Onto the layer's surface small metallic circles of chromium were evaporated. The pin-diode consists of an about $100 \mathrm{~nm}$ thick highly boron doped layer grown onto the $\mathrm{ZnO}$, followed by $800 \mathrm{~nm}$ of slightly boron doped $\mu \mathrm{c}-\mathrm{Si}$. The boron was added in order to compensate the donors present in undoped $\mu \mathrm{c}$-Si. The highly phosphorous doped top layer was about $50 \mathrm{~nm}$ thick. chromium/gold was used as ohmic contact material. Mesa diodes were prepared by wet etching the silicon layers down to the $\mathrm{ZnO}$ contact. 


\section{Results and discussion}

The TEM image in Fig. 1 shows a typical structure of an about $1.2 \mu \mathrm{m}$ thick undoped microcrystalline layer deposited at $225^{\circ} \mathrm{C}$ onto glass. A needlelike structure surrounded by a noncrystalline phase can be seen. The diameter of a single crystal is about $30 \mathrm{~nm}$ whereas the length sometimes exceeds $300 \mathrm{~nm}$. In order to get information about potential contamination from the substrate during deposition the barium content in the silicon layer was measured by EELS. Barium was chosen since Corning 7059 glass contains $25 \%$ bariumoxide. The oxygen content was determined with different electron beam configurations in order to distinguish between the concentration within or at the surface of a single grain and the concentration between the grains in the noncrystalline phase [4]. In order to demonstrate the porosity of the film the carbon concentration arising from the glue was determined. The results of the EELS analysis are shown in Fig. 2. In the porous phase the measured oxygen concentration is 2.15 higher than inside or on the nanocrystals. Due to the preparation of the ultrathin and porous specimen which were exposed to air prior to the transfer into the electron microscope it must be expected that native oxide formation occurs which significantly contributes to the measured oxygen content but was not introduced during the deposition of the $\mu \mathrm{c}-\mathrm{Si}$ layer. All of the examined $\mu \mathrm{c}-\mathrm{Si}$ layers had a porous phase. The volume fraction varied depending on the process conditions between less than $4 \%$ and more than $25 \%$. Optical measurements on nearly all of the samples were dominated by the crystalline properties of the deposited films. Absorption measurements suggest an optical gap close to the one of crystalline silicon (1.12 eV) with a high density of subbandgap states [5]. Raman spectroscopy usually showed a dominant line close to $519 \mathrm{~cm}^{-1}$ which is typical for crystalline silicon and the two reflectance maxima in the photon energy range between $3 \mathrm{eV}$ and $6 \mathrm{eV}$ well known from single crystal surfaces were found in our samples. When exposed to air the lateral conductivity of undoped $\mu \mathrm{c}-$ Si films grown on glass usually exhibits an unstable behaviour which was found to be mainly due to the adsorption and desorption of gases. Some of the films were heat treated in $\mathrm{Ar}, \mathrm{N}_{2}$ and $\mathrm{O}_{2}$ and subsequently heated up under high vacuum conditions. A quadrupol mass spectrometer was used to detect the partial pressure of the gases of interest released from the sample as a function of the temperature. Up to $100^{\circ} \mathrm{C}$ the outgasing of nonreactive $\mathrm{N}_{2}$ and $\mathrm{Ar}$ as well as the outgasing of $\mathrm{O}_{2}$ obeyed an thermal activation law with activation energies, $E_{\text {act }}$, between $0.1 \mathrm{eV}$ and $0.3 \mathrm{eV}$. No significant difference between the use of oxygen and nonreactive gases was found. Above $100^{\circ} \mathrm{C} E_{\text {act }}$ for oxygen changes to values greater than $2 \mathrm{eV}$ indicating that oxidation takes place whereas $E_{\text {act }}$ of $\mathrm{N}_{2}$ and Ar remains unchanged up to $200^{\circ} \mathrm{C}$. The simple structure of undoped $\mu \mathrm{c}-\mathrm{Si}$ deposited on $\mathrm{ZnO}$ exhibited a Schottky type behaviour where the space charge region was located at the $\mathrm{ZnO} / \mu \mathrm{c}-\mathrm{Si}$ interface and the undoped layer was slightly $n$-type. These structures have been used to carry out deep level transient 
spectroscopy (DLTS). Capacitance-voltage and conductance-voltage measurements as a function of the frequency showed that the measured capacitance was given by two capacitors in parallel. One capacitor was determined by the space charge region assumed to be located within the nanocrystals and the other capacitor was independent from the bias voltage. However it obeyed a power law in the frequency dependence which is typical for hopping conduction in highly disordered materials as one will expect for the noncrystalline phase. Despite the results from optical absorption measurements suggesting defect induced subbands rather than distinguishable deep levels three easily resolvable donors with activation energies, $\Delta E$, at $0.027 \mathrm{eV}, 0.185 \mathrm{eV}$ and $0.336 \mathrm{eV}$ were found. The capture cross sections, $\sigma_{\mathrm{n}}$ assuming electronic properties of crystalline silicon were $4 \times 10^{-22} \mathrm{~cm}^{2}$, $4.4 \times 10^{-19} \mathrm{~cm}^{2}$ and $2.5 \times 10^{-18} \mathrm{~cm}^{2}$ respectively. The concentration for all three levels was less than $1 \%$ of the back ground concentration. One acceptor was resolved: $\Delta E=0.104 \mathrm{eV}, \sigma_{\mathrm{p}}=6 \times 10^{-21} \mathrm{~cm}^{2}$. In the pin-device three more shallow donors with $\Delta E<0.140 \mathrm{eV}$ were found. The shallow donor levels probably can be attributed to oxygen. The donor level at $0.336 \mathrm{eV}$ may be caused by tungsten introduced by the filament. However no evidence for iron coming from the chamber walls and zinc diffusion from the substrate was observed. Using conducting tips during the operation of a scanning force microscope (SFM) in the noncontact mode the potential distribution was recorded simultaneously during the topographic measurement on a cross section of the pin-structure. The averaged result of the surface potential and the calculated charge distribution is shown in Fig. 3. Since the measurements were carried out in air the magnitude of the measured values of the surface potential will diverge considerably from those obtained under ideal conditions in high vacuum [6]. It can be seen that the diode rather behaves like two junctions in series (at the $n / i$-interface and at the $i / p^{+}$-transition) than a pin-structure with no charges in the $i$-layer. The $p^{+}-\mathrm{Si} / n-\mathrm{ZnO}$ transition acts as a reverse biased diode in the equivalent circuitry of the structure thus leading to a rather poor photovoltaic output under illumination.

\section{Conclusions}

The investigated microcrystalline silicon layers are composed of a crystalline and a porous, noncrystalline phase. During the layer deposition the porous phase has the capability to efficiently getter contaminants like oxygen and potentially other impurities and dopants. Impurity levels observed in the undoped crystalline needles are in the order of $1 \%$ of the background concentration and can mainly be attributed to oxygen. The electrical properties perpendicular to the substrate's surface, parallel to the main direction of the crystalline needles can be described by the semiconducting properties of the crystals in parallel with a hopping conduction coming from the noncrystalline channels between the crystals. Although the presence of the porous phase during deposition is 
potentially advantageous its parasitic contribution to the output of photovoltaic devices prepared from HWCVD silicon must be further investigated.

\section{Acknowledgements}

This work was supported in part by the Joule-Thermie program of the European Commission, contract number JOR3-CT97-0126.

\section{References}

[1] J. Meier et al., Proceedings of the $2^{\text {nd }}$ World Conference on Photovoltaic Solar Energy Conversion, Vienna, (1998), 375 .

[2] P. Müller et al., Proceedings of the International Conference on Polycrystalline Semiconductors 1998, ed. by

J.H. Werner, H.P. Strunk and H.W.Schock

[3] C. Voz et al., (????) to be published.

[4] M. Stöger, Diplomarbeit am Institut für Angewandte und Technische Physik (TU Wien) 1999, 55-58

[5] A. Breymesser et al., Proceedings of the $2^{\text {nd }}$ World Conference on Photovoltaic Solar Energy Conversion, Vienna, (1998), 1615.

[6] A. Breymesser et al., Solar Energy Materials and Solar Cells, to be published.

\section{Figure captions:}

Fig. 1: TEM image of the cross section of a microcrystalline silicon layer deposited on glass at $\mathrm{T}=225^{\circ} \mathrm{C}$ by hotwire CVD. The substrate is shown in the lower right corner.

Fig. 2: Distribution of oxygen, barium and carbon in the $\mu \mathrm{c}-\mathrm{Si}$ film as detected by EELS.

Fig. 3: Surface potential variation across a pin-structure of $\mu \mathrm{c}-\mathrm{Si}$ and calculated charge distribution. 


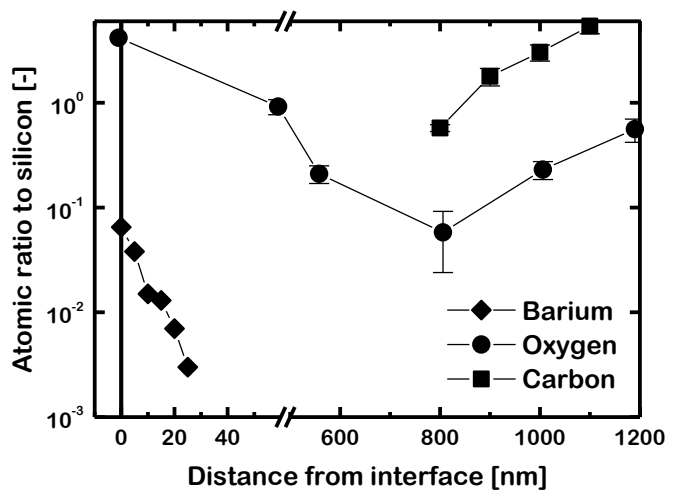

